\title{
Bats, fever and adenopathy - what is the link?
}

\author{
Joshua J Manolakos MD, Mohan Cooray MD, Ameen Patel MB FRCPC, Shariq Haider MD FRCPC
}

\author{
JJ Manolakos, M Cooray, A Patel, S Haider. Bats, fever and \\ adenopathy - what is the link? Can J Infect Dis Med Microbiol \\ $2013 ; 24(1): 35-37$.
}

A case of travel-related, subacute, progressive disseminated histoplasmosis in a nonimmunocompromised individual is described. The present case highlights the environmental exposure to Histoplasma capsulatum in Costa Rica, the diagnostic approach and treatment options, as well as new alternatives for salvage therapy for histoplasmosis infection.

Key Words: Costa Rica; Fever; Histoplasmosis; Posaconazole; Travel

\section{CASE PRESENTATION}

A healthy 20-year-old woman developed headaches, hot flashes, drenching night sweats, rigors and shortness of breath one week after returning from a three-week trip to Costa Rica. Ten days after returning to Canada, blood work revealed a diminished neutrophil count of $1.9 \times 10^{9} / \mathrm{L}$, a borderline thrombocytopenia of $120 \times 10^{9} / \mathrm{L}$ and atypical reactive lymphocytes; a thick and thin smear performed to test for malaria was negative. A chest $\mathrm{x}$-ray was normal and serological testing for Epstein-Barr virus, cytomegalovirus, leptospirosis and Dengue fever was negative.

Three weeks after her return, she experienced persistent fever, malaise, substernal pleuritic chest pain and worsening shortness of breath. A computed tomography (CT) scan of her chest revealed bilateral nodular disease as well as bulky hilar and mediastinal adenopathy. She reported visiting a local barn inhabited by bats while in Costa Rica. Her symptoms, along with the bat exposure and the CT scan results, raised the suspicion of an endemic fungal infection, such as pulmonary histoplasmosis. Noninfectious etiologies, such as lymphoma, remained possible. Cytology and cultures (tuberculosis, fungal and bacterial) performed after bronchoalveolar lavage were negative. An open-lung biopsy was completed six weeks after her return to Canada due to the high suspicion of histoplasmosis, the inability to obtain antigen testing and the limited sensitivity of serological testing early in the course of histoplasmosis. The decision was made by the thoracic surgery service to biopsy two nodules from the right lower lobe because they were more accessible than the mediastinal lymph nodes. The histology from the biopsies revealed necrotizing granulomata, and Grocott stains demonstrated abundant fungal organisms consistent with Histoplasma capsulatum. The mycology culture was negative. An initial diagnosis of pulmonary histoplasmosis was made.

On admission for treatment her chest x-rays demonstrated bilateral lung nodules, patchy bibasilar airspace disease and a cavitating lesion in the right lung (Figure 1). Her aspartate aminotransferase level (48 U/L) and her alanine aminotranferease level (159 U/L) were mildly elevated, and an abdominal ultrasound and an echocardiogram were performed to investigate for extrapulmonary disease. The echocardiogram was normal. Hepatosplenomegaly was present, with a liver and spleen span of $17.4 \mathrm{~cm}$ and $12.4 \mathrm{~cm}$, respectively. A diagnosis of subacute progressive disseminated histoplasmosis (PDH) was made based on the combination of fever, malaise, hepatosplenomegaly, diminished neutrophil and platelet counts, and the biopsy results. Inpatient treatment was initiated with intravenous amphotericin B deoxycholate $40 \mathrm{mg}$ daily for seven days followed by itraconazole $200 \mathrm{mg}$ (capsule formulation) daily.

\section{Le lien entre les chauves-souris, la fièvre et l'adénopathie}

Les chercheurs décrivent un cas d'histoplasmose disséminée subaiguë progressive liée à un voyage chez une personne non immunocompromise. Le présent cas fait ressortir l'exposition environnementale à l'Histoplasma capsulatum au Costa Rica, la démarche diagnostique et les possibilités thérapeutiques, ainsi que de nouvelles possibilités de thérapie de rattrapage de l'infection par l'histoplasmose.

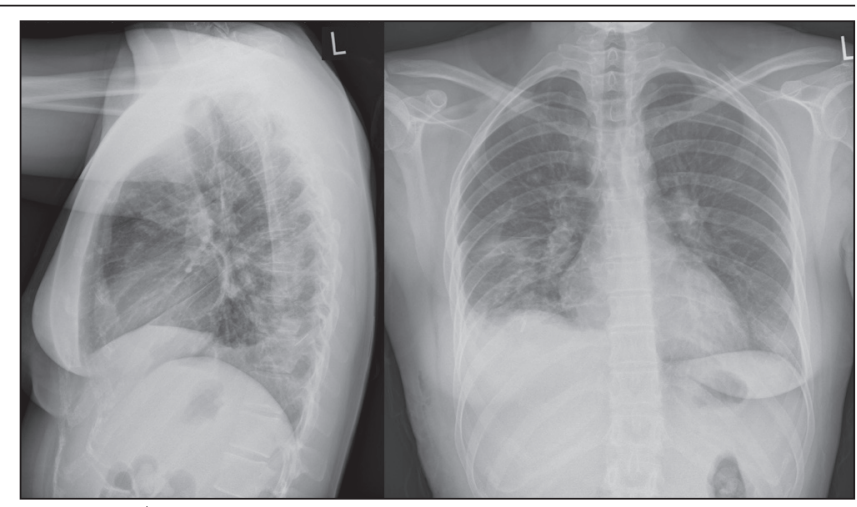

Figure 1) Chest x-rays before treatment demonstrating bilateral lung nodules, patchy bibasilar airspace disease and a cavitating lesion in the mid zone of the right lung

After three months of itraconazole treatment, a repeat CT scan for persistent shortness of breath demonstrated resolution of her hepatomegaly with no change in her splenomegaly. There was a $50 \%$ reduction in the extent of pulmonary nodules and persistent mediastinal and hilar lymphadenopathy. The radiographical findings in addition to the persistent symptoms were considered a partial response to itraconazole according to the European Organisation for Research and Treatment of Cancer criteria (1). Due to the lack of therapeutic drug monitoring for itraconazole levels and partial clinical response, a switch to posaconazole $400 \mathrm{mg}$ twice daily for refractory disease was made. Following six weeks of posaconazole therapy, her respiratory symptoms had resolved and a repeat CT scan demonstrated further interval improvement. After six months of posaconazole therapy she continued to show signs of radiographical improvement (Figure 2) and she successfully completed a one-year course of posaconazole without any recurrence or adverse effects.

Due to the rarity of subacute PDH in healthy individuals, a workup for possible immunodeficiency was completed. She had not experienced any previous infections and did not use intravenous drugs or alcohol. She was not taking immunosuppressive medications and did not have diabetes or kidney disease. She had a slightly low CD4 count of $0.44 \times 10^{9} / \mathrm{L}$. The remainder of her CD3, CD8, CD4/CD8 ratio, B cell testing, quantitative immunoglobulins and chronic granulomatous disease testing was normal. Testing was negative for acute or chronic 


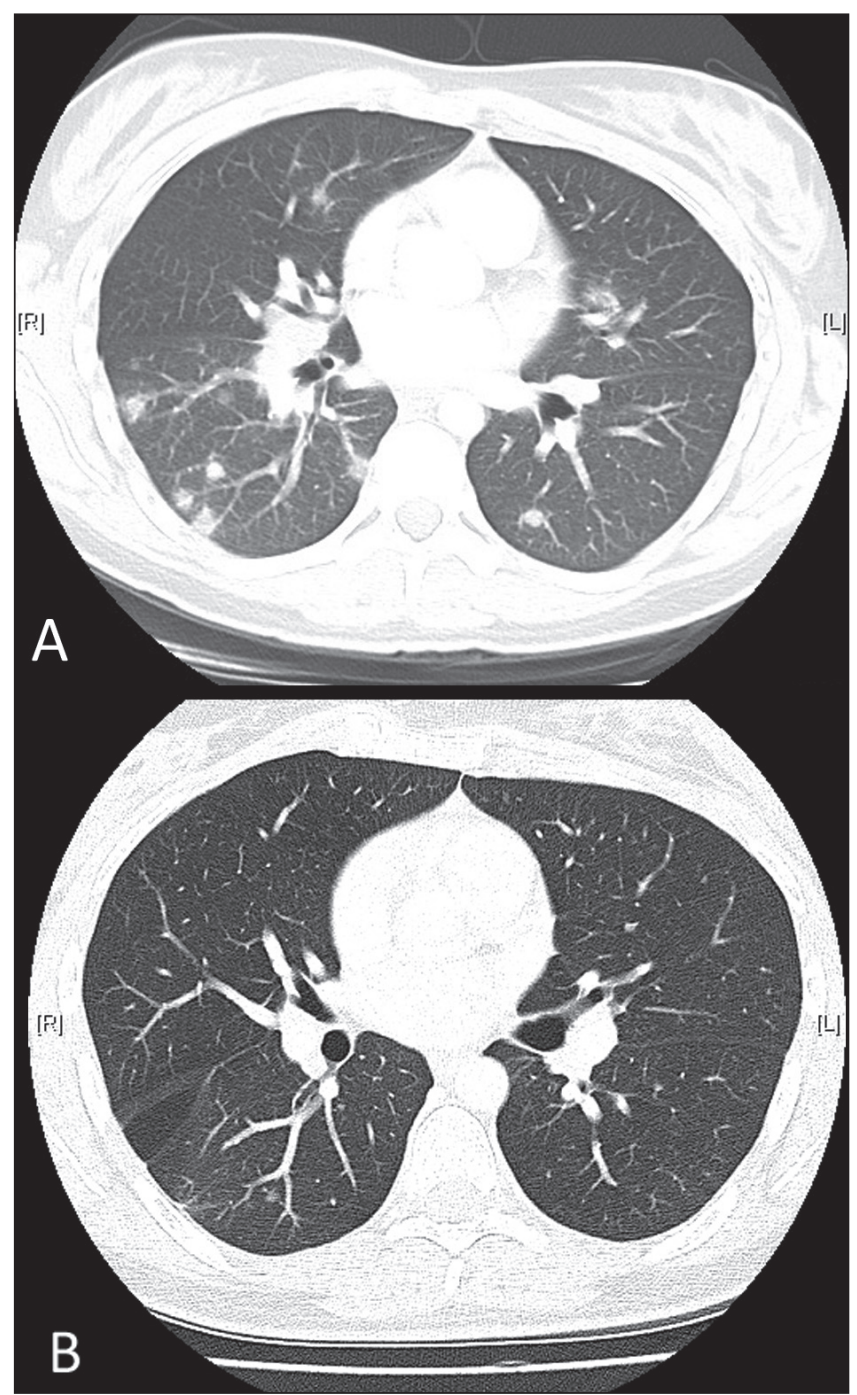

Figure 2) Comparison of a pretreatment computed tomography scan (A) with a scan performed after six months of posaconazole treatment (B)

hepatitis B and hepatitis C infection, and her HIV 1 and HIV 2 ELISA testing were negative. Overall, there was no evidence of either primary or secondary immunodeficiency.

\section{DISCUSSION}

Histoplasmosis is a fungal infection caused by $\mathrm{H}$ capsulatum (2). $\mathrm{H}$ capsulatum is a thermally dimorphic fungus that grows in its mycelial phase in soil (3). Its growth is enhanced in soils enriched by avian and bat excreta (3-6). Endemic areas of histoplasmosis include most of Latin America, eastern Asia, Africa, Australia and the eastern half of the United States, including the Mississippi and Ohio River Valleys $(3,4)$. In Canada, it is found predominantly in the central provinces along the St Lawrence River, with isolated reports from Atlantic provinces, Northern Territories and Alberta (7-9). Histoplasmosis rarely occurs in individuals living outside of endemic areas unless it is 'imported' from an endemic area (10).

The patient presented lived in an urban community in southern Ontario. It can be reasonably postulated that she developed histoplasmosis during her travels in Costa Rica because her symptoms began within a week of returning to Ontario. Although histoplasmosis is a well described travel illness (11), there are few reported cases of travelrelated histoplasmosis originating from Costa Rica $(5,12)$ where subjects were directly exposed while exploring caves $(5,12)$.
The diagnosis of histoplasmosis can be made via cultures, histopathology, antigen detection or serology (10). Culture, histopathology and antigen detection are more useful when the fungal burden is high (as in acute diffuse pulmonary histoplasmosis and PDH), while serological tests for antibodies are of higher yield when the fungal burden is lower (as in subacute pulmonary histoplasmosis). Urine antigen testing has the highest sensitivity, although any body fluid may be used (10). In the present case, urine antigen testing would have corroborated the clinical suspicion of $\mathrm{PDH}$; however, at the time, testing was not available at our local or public health microbiology laboratories. This, along with the negative cultures and a high suspicion of histoplasmosis, led to the decision to pursue an open-lung biopsy to confirm the diagnosis.

$\mathrm{PDH}$ is most commonly seen in immunocompromised individuals, particularly in patients with impaired cell-mediated immunity secondary to immunosuppressive medications, HIV infection and lymphoma $(4,10)$. Given the negative workup for immunodeficiency, the patient represented a small minority of patients with normal immune function who develop PDH $(10,13,14)$. It remains possible, however, that she has an unidentified immunodeficiency.

The management of patients with histoplasmosis is dependent on the severity and form of histoplasmosis. Guidelines recommend treatment for severe or moderately severe acute pulmonary, chronic pulmonary, disseminated and central nervous system histoplasmosis $(15,16)$. The initial regimen of amphotericin and itraconazole used in the current case has an average success rate of $80 \%$ to $100 \%$ (16). The optimal salvage therapy in refractory histoplasmosis has yet to be established (16).

Voriconazole and posaconazole have been successfully used for salvage therapy, although posaconazole appears to be more active in vitro and in experimental infection (17). Posaconazole is an extendedspectrum triazole antifungal agent, which has been used successfully for the salvage treatment of pulmonary and PDH using doses of $400 \mathrm{mg}$ twice daily $(18,19)$. The largest published case series of salvage treatment with posaconazole included six patients, five of whom experienced disseminated disease, and five of six were immunocompromised (19). Two of these patients were started on posaconazole due to treatment failure with other antifungal medications, including amphotericin B, itraconazole and voriconazole. The other cases were switched due to antifungal intolerance. Clinical improvement was apparent in all six patients within one month of the initiation of posaconazole therapy (19). The present case demonstrated the successful use of posaconazole for the salvage treatment of subacute PDH without any adverse effects.

\section{CONCLUSION}

There are several clinically relevant and important elements to be learned from the present case. It highlights the importance of a detailed travel history when attempting to uncover potential exposures, and to consider histoplasmosis in febrile travellers returning from Central America. Although it is rare, the diagnosis of PDH must still be considered in immunocompetent patients with histoplasmosis because the diagnosis can alter the type and duration of antifungal treatment. Finally, the present case further demonstrates that the use of posaconazole is a safe and effective strategy for the treatment of histoplasmosis.

\section{REFERENCES}

1. Segal BH, Herbrecht R, Stevens DA, et al. Defining responses to therapy and study outcomes in clinical trials of invasive fungal diseases: Mycoses Study Group and European Organization for Research and Treatment of Cancer consensus criteria. Clin Infect Dis 2008;47:674-83.

2. Fauci AS, Graw-Hill C. Harrison's Principles of Internal Medicine. Fauci AS, Braunwald E, Kasper DL, et al, eds. 17th edn. New York: McGraw-Hill Medical; 2008.

3. Wang TL, Cheah JS, Holmberg K. Case report and review of disseminated histoplasmosis in South-East Asia: Clinical and epidemiological implications. Trop Med Int Health 1996;1:35-42.

4. Goldman M, Johnson PC, Sarosi GA. Fungal pneumonias: The endemic mycoses. Clin Chest Med 1999;20:507-19. 
5. Lyon GM, Bravo AV, Espino A, et al. Histoplasmosis associated with exploring a bat-inhabited cave in Costa Rica, 1998-1999. Am J Trop Med Hyg 2004;70:438-42.

6. Norman FF, Martin-Davila P, Fortun J, et al. Imported histoplasmosis: Two distinct profiles in travelers and immigrants. J Travel Med 2009;16:258-62.

7. Locally acquired histoplasmosis cluster, Alberta, 2003. Can Commun Dis Rep 2005;31:255-8.

8. Wheat J. Histoplasmosis: Experience during outbreaks in Indianapolis and review of the literature. Medicine (Baltimore) 1997;76:339-54

9. Anderson H, Honish L, Taylor G, et al. Histoplasmosis cluster, golf course, Canada. Emerg Infect Dis 2006;12:163-5.

10. Wheat LJ. Histoplasmosis: A review for clinicians from non-endemic areas. Mycoses 2006;49:274-82.

11. Chiller T. Traveler's Health - Yellow Book. Centers for Disease Control and Prevention 2009. <www.nc.cdc.gov/travel/ yellowbook/2010/chapter-5/histoplasmosis.aspx $>$ (Accessed January 25, 2011).

12. Anonymous. Cave-associated histoplasmosis - Costa Rica. Morb Mortal Wkly Rep 1988;37:312-3.

13. Harten P, Euler HH, Wolf E, Delling G, Loffler H. Disseminated histoplasmosis in a non-immunocompromised host. Clin Investig 1994;72:878-82.
14. Ohji G, Kikuchi K, Inoue K, et al. Progressive disseminated histoplasmosis in an immunocompetent patient as an underrecognized imported mycosis in Japan. J Infect Chemother 2010;16:443-5.

15. Wheat J, Sarosi G, McKinsey D, et al. Practice guidelines for the management of patients with histoplasmosis. Infectious Diseases Society of America. Clin Infect Dis 2000;30:688-95.

16. Wheat LJ, Freifeld AG, Kleiman MB, et al. Clinical practice guidelines for the management of patients with histoplasmosis: 2007 update by the Infectious Diseases Society of America. Clin Infect Dis 2007;45:807-25.

17. Connolly P, Wheat J, Schnizlein-Bick C, et al. Comparison of a new triazole antifungal agent, Schering 56592, with itraconazole and amphotericin B for treatment of histoplasmosis in immunocompetent mice. Antimicrob Agents Chemother 1999;43:322-8

18. Clark B, Foster R, Tunbridge A, Green S. A case of disseminated histoplasmosis successfully treated with the investigational drug posaconazole. J Infect 2005;51:e177-e80.

19. Restrepo A, Tobon A, Clark B, et al. Salvage treatment of histoplasmosis with posaconazole. J Infect 2007;54:319-27. 


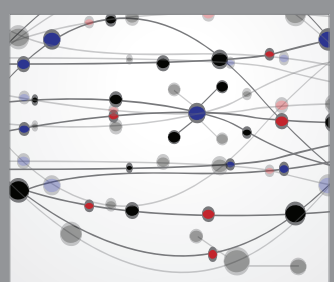

The Scientific World Journal
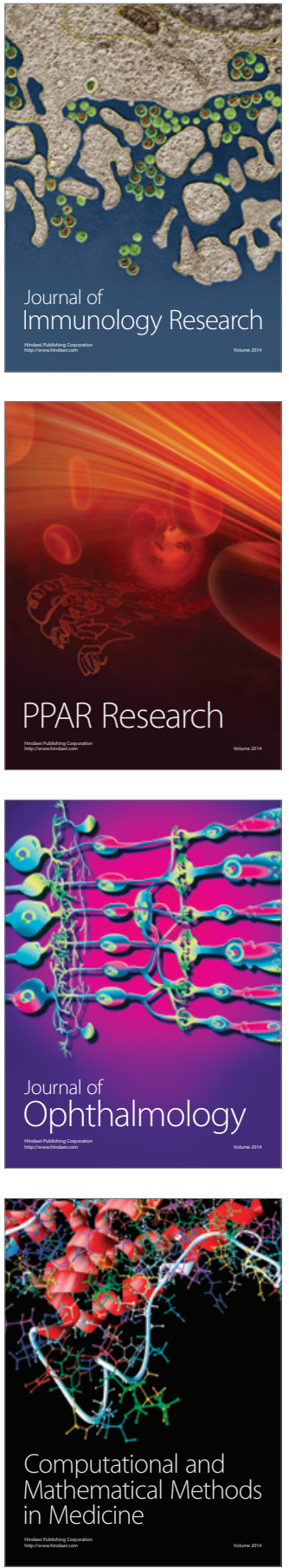

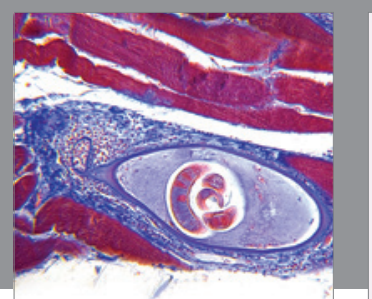

Gastroenterology Research and Practice

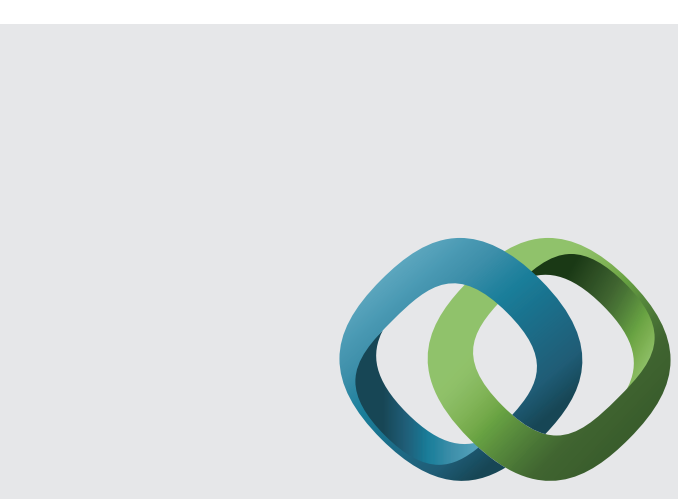

\section{Hindawi}

Submit your manuscripts at

http://www.hindawi.com
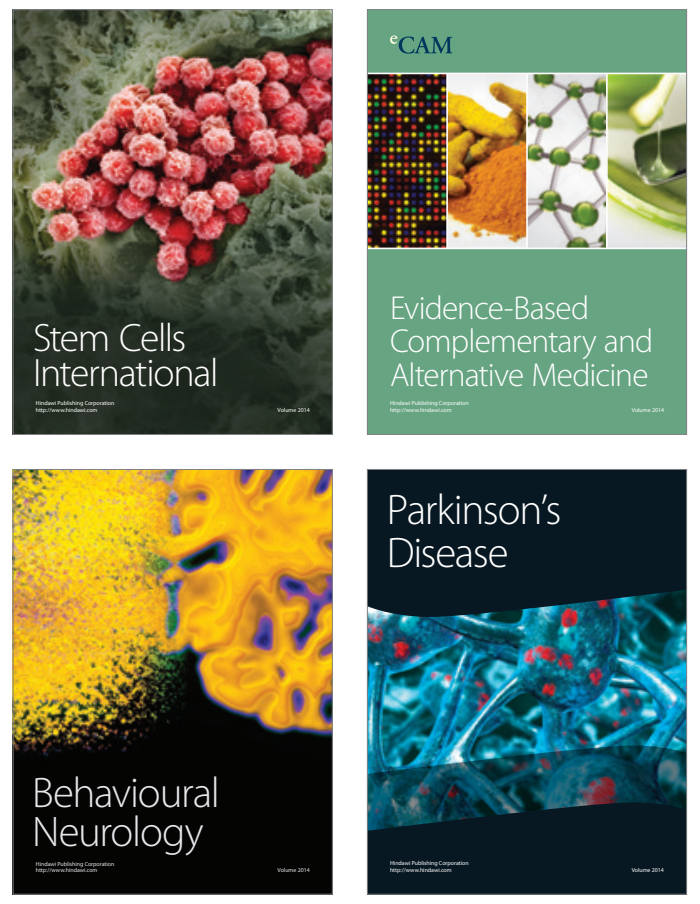
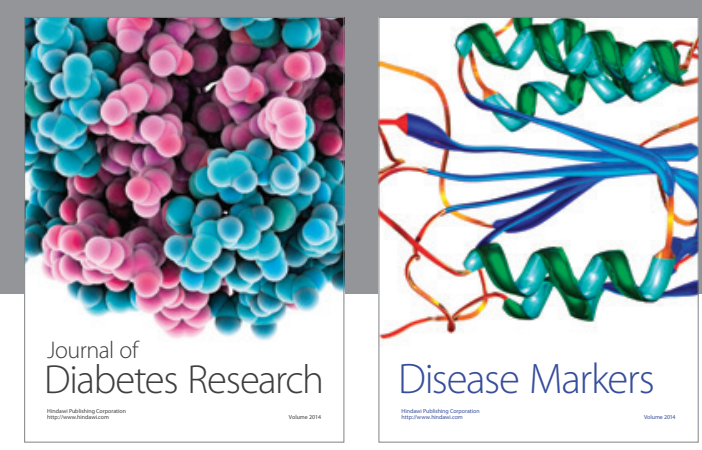

Disease Markers
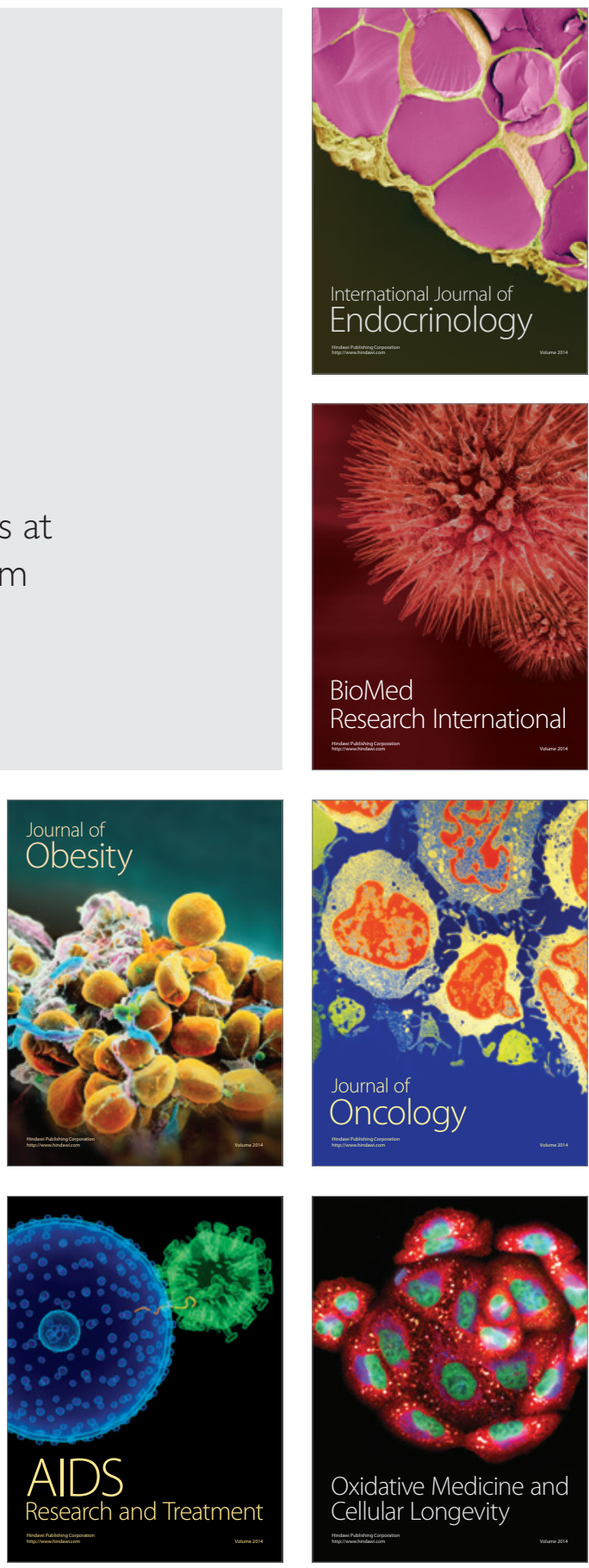